\title{
The association between PD-L1 and EGFR status and the prognostic value of PD-L1 in advanced non-small cell lung cancer patients treated with EGFR-TKIs
}

\author{
Yanna Tang ${ }^{1,2,3, *}$, Wenfeng Fang ${ }^{1,2,3, *}$, Yaxiong Zhang ${ }^{1,2,3, *}$, Shaodong Hong ${ }^{1,2,3}$, \\ Shiyang Kang ${ }^{1,2,3}$, Yue Yan ${ }^{1,2,3}$, Nan Chen ${ }^{1,2,3}$, Jianhua Zhan ${ }^{1,2,3}$, Xiaobo He ${ }^{1,2,3}$, Tao \\ Qin $^{1,2,3}$, Ge Li ${ }^{4}$, Wenyi Tang ${ }^{5}$, Peijian Peng ${ }^{5}$ and Li Zhang ${ }^{1,2,3}$ \\ ${ }^{1}$ Department of Medical Oncology, Sun Yat-sen University Cancer Center, Guangzhou, China \\ 2 State Key Laboratory of Oncology in South China, Guangzhou, China \\ ${ }^{3}$ Collaborative Innovation Center for Cancer Medicine, Guangzhou, China \\ ${ }^{4}$ Key Laboratory for Stem Cells and Tissue Engineering, Sun Yat-sen University, Guangzhou, China \\ ${ }^{5}$ Department of Medical Oncology, The Fifth Affiliated Hospital of Sun Yat-Sen University, Zhu Hai, China \\ * These authors have contributed equally to this work \\ Correspondence to: Li Zhang, email: zhanglib@mail.sysu.edu.cn
}

Peijian Peng, email: pengpjian@163.com

Keywords: NSCLC, PD-L1, EGFR status, TKI, prognosis

Received: January 08, $2015 \quad$ Accepted: March 01, $2015 \quad$ Published: March 29, 2015

This is an open-access article distributed under the terms of the Creative Commons Attribution License, which permits unrestricted use, distribution, and reproduction in any medium, provided the original author and source are credited.

\section{ABSTRACT}

Backgrounds: Recent clinical trials have shown that immune-checkpoint blockade yields remarkable response in a subset of non-small cell lung cancer (NSCLC) patients. However, few studies directly focus on the association between epidermal growth factor receptor (EGFR) mutational status and programmed cell death-ligand 1 (PDL1) expression. We examined whether PD-L1 is related to clinicopathologic factors and prognosis in patients with advanced NSCLC treated with EGFR-tyrosine kinase inhibitors (EGFR-TKIs).

Methods: One-hundred and seventy patients with advanced NSCLC were explored. Paraffin-embedded tumour sections were stained with PD-L1 antibody. EGFR mutation was examined by fluorescent quantitative polymerase chain reaction (PCR). The correlations between PD-L1 expression and EGFR status and survival parameters were analyzed.

Results: The overall frequency of PD-L1 over-expression was 65.9\% (112/170). In lung adenocarcinoma, PD-L1 tended to be associated with mutant EGFR (PD-L1 overexpression in mutant and wild-type EGFR, 64/89 (71.9\%) vs. 32/56 (57.1\%), respectively; $\mathrm{p}=0.067$ ). Subgroup analyses showed that high PD-L1 expression was associated with significantly shorter overall survival (OS) in EGFR wild-type patients $(p=0.029)$ but not in EGFR mutant patients $(p=0.932)$ treated with EGFR-TKIs. Even more, for EGFR mutant patients, higher expression of PD-L1 might only signal better outcome with TKIs.

Conclusions: High PD-L1 expression was likely to be associated with the presence of EGFR mutation in advanced lung adenocarcinoma. For EGFR wild-type patients, the PD-L1 over expression can be considered as a poor prognostic indicator of OS. 


\section{INTRODUCTION}

Lung cancer, especially non-small cell lung cancer (NSCLC), is currently the leading cause of cancer-related death worldwide [1]. Recent advancements in targeted therapy have led to a major paradigm shift in the treatment of advanced NSCLC [2]. Molecularly targeted drugs such as erlotinib and gefitinib have thus greatly improved the clinical outcome of advanced NSCLC patients harboring sensitive epidermal growth factor receptor (EGFR) gene mutations [3]. Two major types of EGFR kinase mutations include exon-19 deletions and L858R mutation in exon $21[4,5]$.

Programmed death 1 (PD-1) is a co-inhibitory receptor expressed on the membrane of activated $\mathrm{T}$ and B cells [6], which plays a crucial role in tumor immune escape $[7,8]$. Programmed cell death-ligand 1 (PD-L1) is the major ligand for PD-1 and is expressed in a variety of cancers $[7,9]$. PD-L1 has been shown to be involved in the negative regulation of immune response through PD-1 receptor and has been thought to be an important strategy for cancer cells to evade host immune surveillance. Cancer cells expressing PD-L1 have been shown to increase apoptosis of antigen-specific human T-cell clones and to inhibit CD4 and CD8 T-cell activation in vitro [10-12].

Currently, some studies demonstrated that PD-L1 was expressed in $19.63 \%-65.38 \%$ of NSCLC [2, 13-16]. Several studies suggested that PD-L1 expression portended inconsistent survival outcomes [17]. For example, a study showed that tumor with a high level of PD-L1 expression was associated with significantly shorter overall survival (OS) in NSCLC patients [2], while another report showed positive PD-L1 was significantly associated with better survival outcome [15]. Now, the molecular regulatory mechanism of PD-L1 isn't comprehensive enough, though two studies found that mutant EGFR could induce PD-L1 expression in vitro and vivo, and EGFR tyrosine kinase inhibitors (EGFR-TKIs) could down-regulate PDL1 expression $[2,16]$. It is therefore of significance to study the association between EGFR driver mutation and PD-L1. Even more, the incidence of EGFR mutations is higher in East Asian patients than in Caucasian patients (30\% versus $8 \%$ ) [4], and there are no studies examining the relationship between PD-L1 expression and efficacy in patients treated with EGFR-TKIs in China. Therefore,we tried to investigate the impact of PD-L1 expression on EGFR-TKIs' efficacy and prognosis in Chinese advanced NSCLC patients, emphasizing on the EGFR mutational status.

\section{RESULTS}

\section{Patient characteristics}

A total of 170 eligible patients with advanced NSCLC were included in the present study (Figure 1). The mean age at diagnosis was 57.09 years (range, $32-80$ years) (Table 1). Seventy-seven $(45.3 \%)$ of the patients were female and ninety-three $(54.7 \%)$ were male. Fiftyseven $(33.5 \%)$ patients were smokers. Nine $(5.3 \%)$ patients and $161(94.7 \%)$ patients were diagnosed at stage IIIB and stage IV, respectively. In the current research, 99 patients harbored EGFR mutation and the remaining 71 patients were EGFR wild type. With regard to EGFR mutation status, 40 patients harbored a deletion in exon 19 and 47 patients had an L858R missense mutation in exon 21. 12 were uncommon mutation.

\section{Correlations between PD-L1 expression and baseline characteristics}

Immunohistochemical staining for PD-L1 was found at the membrane or in the cytoplasm (or both) of tumor cells (Figure 2A). As shown in Table 1, PD-L1 is over expressed in $65.9 \%(112 / 170)$ of advanced NSCLC patients. The relationship between PD-L1 expression and age, gender, histopathological type, tumor stage and EGFR mutational status was not significant, except for the line of EGFR TKIs $(P=0.041)$. However, in subgroup of lung adenocarcinoma, there was a borderline difference between PD-L1 expression level and EGFR mutational status (32/56 (57.1\%) for wild type and 64/89 (71.9\%) for mutant type, respectively, $\mathrm{p}=0.067$ ).

\section{Relationships between PD-L1 expression and the EGFR-TKIs' efficacy}

The association between the efficacy of EGFR-TKIs with PD-L1 expression as well as other clinicpathologic factors in advanced NSCLC patients was summarized in Table 2. There was no significant relationship between objective response rate (ORR) and PD-L1 expression, as well as age, gender, histopathological type, stage and TKI line. However, patients with mutant EGFR had better ORR than those with wild-type EGFR (odds ratio (OR), 0.266 ; $95 \%$ confidence interval $(95 \% \mathrm{CI}), 0.114$ to $0.621 ; \mathrm{p}=0.002)$ and non-smokers also had higher ORR than smokers did (OR, 4.667; 95\% CI, 1.716 to 12.693 ; $\mathrm{p}=0.003)$. These results were in accordance with the results of multivariate analysis. Besides, we examined the association between a variety of factors and disease control rate (DCR). We found that there was no significant difference between DCR and PD-L1 status (OR, 0.783; 
Table 1: Clinicopathological characteristics of patients with NSCLC $(n=170)$ and their relationship with PD-L1 expression.

\begin{tabular}{|c|c|c|c|c|c|c|c|c|c|}
\hline \multirow{2}{*}{ Parameter } & \multicolumn{4}{|c|}{ PD-L1 positive } & \multicolumn{4}{|c|}{ PD-L1 negative } & \multirow[b]{2}{*}{ P-value } \\
\hline & total & PR & SD & PD & total & PR & SD & PD & \\
\hline \multicolumn{10}{|l|}{ Age } \\
\hline$\leq 50 \mathrm{y}$ & 28 & 6 & 17 & 5 & 17 & 3 & 9 & 5 & 0.546 \\
\hline$>50 y$ & 84 & 19 & 51 & 14 & 41 & 12 & 22 & 7 & \\
\hline \multicolumn{10}{|l|}{ Gender } \\
\hline female & 56 & 13 & 38 & 5 & 21 & 8 & 11 & 2 & 0.087 \\
\hline male & 56 & 12 & 30 & 14 & 37 & 7 & 20 & 10 & \\
\hline \multicolumn{10}{|l|}{ Smoking } \\
\hline no & 77 & 21 & 50 & 6 & 36 & 14 & 15 & 7 & 0.382 \\
\hline yes & 35 & 4 & 18 & 13 & 22 & 1 & 16 & 5 & \\
\hline \multicolumn{10}{|l|}{ Pathology } \\
\hline $\mathrm{ADC}$ & 96 & 24 & 58 & 14 & 49 & 14 & 25 & 10 & 0.830 \\
\hline non-ADC & 16 & 1 & 10 & 5 & 9 & 1 & 6 & 2 & \\
\hline \multicolumn{10}{|l|}{ Stage } \\
\hline IIIB & 7 & 1 & 5 & 1 & 2 & 0 & 2 & 0 & 0.680 \\
\hline IV & 105 & 24 & 63 & 18 & 56 & 15 & 29 & 12 & \\
\hline \multicolumn{10}{|l|}{ EGFR status } \\
\hline wild type & 42 & 5 & 21 & 16 & 29 & 3 & 16 & 10 & 0.330 \\
\hline exon 19 del & 26 & 6 & 19 & 1 & 14 & 7 & 6 & 1 & \\
\hline exon $21 \mathrm{~L} 858 \mathrm{R}$ & 35 & 11 & 23 & 1 & 12 & 4 & 7 & 1 & \\
\hline unknown classical & 9 & 3 & 5 & 1 & 3 & 1 & 2 & 0 & \\
\hline \multicolumn{10}{|l|}{ EGFR-TKI } \\
\hline 1st line & 61 & 16 & 37 & 8 & 22 & 8 & 12 & 2 & 0.041 \\
\hline$\geq 2$ nd line & 51 & 9 & 31 & 11 & 36 & 7 & 19 & 10 & \\
\hline \multicolumn{10}{|l|}{ Pathology $=$ ADC } \\
\hline EGFR wild type & 32 & 4 & 15 & 13 & 24 & 3 & 13 & 8 & 0.067 \\
\hline EGFR mutation & 64 & 20 & 43 & 1 & 25 & 11 & 12 & 2 & \\
\hline
\end{tabular}

Notes: $\mathrm{H}$-score $\geq 5$ was used as cut-off value to distinguish positive and negative expression of PD-L1.

Abbreviations: PD-L1, programmed cell death-ligand 1; ADC, adenocarcinoma; non-ADC, non-adenocarcinoma;

EGFR, epidermal growth factor receptor; EGFR-TKIs, EGFR-tyrosine kinase inhibitors.

$95 \% \mathrm{CI}, 0.350$ to $1.751 ; \mathrm{p}=0.551)$. Whereas, DCR was significantly higher in women than that in men (OR, $3.478 ; 95 \% \mathrm{CI}, 1.407$ to $8.600 ; \mathrm{P}=0.007)$, in neversmokers than that in smokers (OR, 3.55; $95 \%$ CI, 1.589 to 7.930; $\mathrm{P}=0.002)$, and in those with EGFR mutation than that in those EGFR with wild type (OR, 0.092; 95\% CI, 0.033 to $0.256 ; \mathrm{P}<0.001$ ) (Table 2 ). And the multivariate analysis revealed that EGFR mutation positivity was an independent factor (OR, 0.113 ; 95\% CI, 0.038 to 0.342 ; $\mathrm{P}=0.007)$. We further divided patients into two subgroups: (I) EGFR wild type ( $n=71)$ and (II) EGFR mutant $(n=99)$. No significant differences in two subgroups were found between PD-L1 expression and ORR (OR, 0.854; 95\% CI,
0.187 to $3.891 ; \mathrm{P}=0.838$ and $\mathrm{OR}, 1.765 ; 95 \% \mathrm{CI}, 0.715$ to $4.353 ; \mathrm{P}=0.218$ for group I and group II, respectively), as well as PD-L1 expression and DCR (OR, 1.169; 95\% CI, 0.436 to $3.137 ; \mathrm{P}=0.756$ and $\mathrm{OR}, 0.604 ; 95 \% \mathrm{CI}, 0.096$ to $3.822 ; \mathrm{P}=0.593$ for group I and group II, respectively).

\section{Survival analyses is in NSCLC patients}

The median overall survival of the whole patients was 39.9 months. Kaplan-Meier analysis revealed that overall patients with positive PD-L1 and negative PDL1 expression had no significant difference in OS and progression-free survival (PFS) (Figure 3A, 3D).To 
Table 2: The association between PD-L1 expression and EGFR-TKIS' efficacy in univariate and multivariate logistic regression analysis \#.

\begin{tabular}{|c|c|c|c|c|c|c|c|c|c|c|c|c|c|c|c|c|c|c|}
\hline \multirow{3}{*}{ Parameter } & \multicolumn{9}{|c|}{ ORR } & \multicolumn{9}{|c|}{ DCR } \\
\hline & \multicolumn{6}{|c|}{ Univariate analysis } & \multicolumn{3}{|c|}{ Multivariate analysis } & \multicolumn{6}{|c|}{ Univariate analysis } & \multicolumn{3}{|c|}{ Multivariate analysis } \\
\hline & & PR & SD+PD & OR & $95 \% \mathrm{CI}$ & P-value & OR & $95 \% \mathrm{CI}$ & P-value & & PD & $\mathrm{PR}+\mathrm{SD}$ & OR & $95 \% \mathrm{CI}$ & P-value & OR & $95 \% \mathrm{CI}$ & P-value \\
\hline \multicolumn{19}{|l|}{ Age } \\
\hline$\leq 50$ & & 9 & 36 & 0.758 & $0.329-1.748$ & 0.516 & 0.663 & $0.264-1.666$ & 0.382 & & 10 & 35 & 0.707 & $0.304-1.645$ & 0.421 & 0.495 & $0.181-1.358$ & 0.172 \\
\hline$>50$ & 1 & 31 & 94 & & & & & & & 1 & 21 & 104 & & & & & & \\
\hline \multicolumn{19}{|l|}{ Gender } \\
\hline female & & 21 & 56 & 1.461 & $0.717-2.974$ & 0.296 & 0.541 & $0.220-1.331$ & 0.181 & & 7 & 70 & 3.478 & $1.407-8.600$ & 0.007 & 1.899 & $0.528-6.837$ & 0.326 \\
\hline male & 1 & 19 & 74 & & & & & & & 1 & 24 & 69 & & & & & & \\
\hline \multicolumn{19}{|l|}{ Smoking } \\
\hline no & & 35 & 78 & 4.667 & $1.716-12.693$ & 0.003 & 5.776 & $1.763-18.863$ & 0.004 & & 13 & 100 & 3.55 & $1.589-7.930$ & 0.002 & 1.942 & $0.612-6.154$ & 0.260 \\
\hline yes & 1 & 5 & 52 & & & & & & & 1 & 18 & 39 & & & & & & \\
\hline \multicolumn{19}{|l|}{ Pathology } \\
\hline $\mathrm{ADC}$ & & 38 & 107 & 4.084 & $0.919-18.150$ & 0.064 & 3.834 & $0.793-18.538$ & 0.095 & & 24 & 121 & 1.961 & $0.738-5.207$ & 0.177 & 1.348 & $0.439-4.142$ & 0.602 \\
\hline non-ADC & 1 & 2 & 23 & & & & & & & 1 & 7 & 18 & & & & & & \\
\hline \multicolumn{19}{|l|}{ Stage } \\
\hline IIIB & & 1 & 8 & 0.391 & $0.047-3.225$ & 0.383 & 0.206 & $0.023-1.877$ & 0.161 & & 1 & 8 & 1.832 & $0.221-15.208$ & 0.575 & 0.576 & $0.046-7.175$ & 0.668 \\
\hline IV & 1 & 39 & 122 & & & & & & & 1 & 30 & 131 & & & & & & \\
\hline \multicolumn{19}{|l|}{ EGFR } \\
\hline wild type & & 8 & 63 & 0.266 & $0.114-0.621$ & 0.002 & 0.351 & $0.137-0.904$ & 0.030 & & 26 & 42 & 0.092 & $0.033-0.256$ & $<0.001$ & 0.113 & $0.038-0.342$ & $<0.001$ \\
\hline mutation & 1 & 32 & 67 & & & & & & & 1 & 5 & 97 & & & & & & \\
\hline \multicolumn{19}{|l|}{ EGFR-TKI } \\
\hline 1st line & & 24 & 59 & 1.805 & $0.878-3.711$ & 0.108 & 1.863 & $0.798-4.349$ & 0.150 & & 10 & 73 & 2.323 & $1.020-5.291$ & 0.045 & 1.394 & $0.525-3.702$ & 0.506 \\
\hline$\geq 2$ nd line & 1 & 16 & 71 & & & & & & & 1 & 21 & 66 & & & & & & \\
\hline \multicolumn{19}{|l|}{ PD-L1 } \\
\hline negative & & 15 & 43 & 1.214 & $0.581-2.537$ & 0.606 & 1.674 & $0.711-3.939$ & 0.238 & & 12 & 46 & 0.783 & $0.350-1.751$ & 0.551 & 1.109 & $0.445-2.764$ & 0.824 \\
\hline positive & 1 & 25 & 87 & & & & & & & 1 & 19 & 93 & & & & & & \\
\hline \multicolumn{19}{|l|}{ EGFR wild-type } \\
\hline \multicolumn{19}{|l|}{ subgroup } \\
\hline PD-Ll negative & & 3 & 26 & 0.854 & $0.187-3.891$ & 0.838 & & & & & 10 & 19 & 1.169 & $0.436-3.137$ & 0.756 & & & \\
\hline PD-L1 positive & 1 & 5 & 37 & & & & & & & 1 & 16 & 26 & & & & & & \\
\hline \multicolumn{19}{|l|}{ EGFR mutant } \\
\hline \multicolumn{19}{|l|}{ subgroup } \\
\hline PD-L1 negative & & 12 & 17 & 1.765 & $0.715-4.353$ & 0.218 & & & & & 2 & 27 & 0.604 & $0.096-3.822$ & 0.593 & & & \\
\hline PD-LI positive & 1 & 20 & 50 & & & & & & & 1 & 3 & 67 & & & & & & \\
\hline
\end{tabular}

\# A total of 170 non-small cell lung cancer patients were included.

Abbreviations: ORR, objective response rate; DCR, disease control rate; PR, partial response; SD, stable disease; $\mathrm{PD}$, progressive disease ; OR, odd ratio; 95\% CI, 95\% confidence intervals; ADC, adenocarcinoma; non-ADC, nonadenocarcinoma; EGFR, epidermal growth factor receptor; EGFR-TKI, EGFR-tyrosine kinase inhibitor; PD-L1, programmed cell death-ligand 1.

investigate the PD-L1's clinical significance, we further divided the patients into two groups: EGFR mutation and EGFR wild type. In EGFR mutation group, PFS and OS of patients with positive PD-L1 tended to be longer than patients with negative PD-L1, although statistical significance was not achieved (Figure 3B, 3E). In EGFR wild type group, negative $\mathrm{PD}-\mathrm{L} 1$ patients have longer OS than positive-PD-L1 patients $(\mathrm{P}=0.029)$ (Figure $3 \mathrm{C}$ ), while no significant difference in PFS was observed (Figure 3F). Exploratory analysis was done to validate the prognostic role of PD-L1 in subgroups defined by age, sex, smoking, pathology, stage of disease, EGFR mutation, and EGFRTKI's lines (Figure 4). We found that patients with positive PD-L1 expression had signal better prognosis in EGFR mutation subgroup, contrary to EGFR wild-type group. To determine the prognostic value of PD-L1 expression, we carried out univariate and multivariate analyses using the Cox regression model. For PFS and OS, EGFR mutation remained the independent factor for better prognosis (hazard ratio (HR), 0.419; 95\% CI, 0.262-0.672; $\mathrm{P}<0.001$ for PFS and HR, 0.499; 95\% CI, 0.264-0.942; $\mathrm{P}=0.032$ for OS, respectively) (Table 3 ). In subgroup multivariate analysis, we found that the high level PD-L1 can be considered as a poor prognostic indicator of OS for EGFR wild-type patients (HR, 3.738; 95\% CI, 1.341-10.419; $\mathrm{P}=0.012)$ (Supplementary table 1$)$.

\section{DISCUSSION}

NSCLC is partially characterized by driver mutation-defined molecular subsets, each with distinct clinicopathologic features and potentials for targeted therapies. In the present study, we found that PD-L1 was over-expressed in $65.9 \%$ of advanced NSCLC samples and positive PD-L1 tended to be associated with EGFR mutation. We also revealed that there was no significant correlation between expression of PD-L1 and curative effect of EGFR TKIs (ORR and DCR). In EGFR mutation group, PFS and OS of patients positive for PD-L1 tended to be signal better than that of patients who were negative, although statistical significance was not achieved. For patients with wild type EGFR, PD-L1-negative NSCLC patients had longer overall survival than PD-L1-positive ones.

In previous studies, researchers have demonstrated that activation of the EGFR pathway induced PD-L1 
Table 3: Univariate and multivariate analyses of OS and PFS in NSCLC patients.

\begin{tabular}{|c|c|c|c|c|c|c|c|c|c|c|c|c|c|c|c|c|}
\hline \multirow{3}{*}{ Parameter } & \multicolumn{8}{|c|}{ OS } & \multicolumn{8}{|c|}{ PFS } \\
\hline & & \multirow[b]{2}{*}{$\mathrm{N}$} & \multirow[b]{2}{*}{ HR } & \multicolumn{2}{|c|}{ Univariate analysis } & \multicolumn{3}{|c|}{ Multivariate analysis } & & \multirow[b]{2}{*}{$\mathrm{N}$} & \multicolumn{3}{|c|}{ Univariate analysis } & \multicolumn{3}{|c|}{ Multivariate analysis } \\
\hline & & & & $95 \% \mathrm{CI}$ & P-value & HR & $95 \% \mathrm{CI}$ & P-value & & & HR & $95 \% \mathrm{CI}$ & P-value & HR & $95 \% \mathrm{CI}$ & P-value \\
\hline \multicolumn{17}{|l|}{ Age } \\
\hline$\leq 50 \mathrm{y}$ & 1 & 45 & & & & & & & 1 & 45 & & & & & & \\
\hline$>50 y$ & & 125 & 2.552 & $1.082-6.019$ & 0.032 & 2.602 & $1.078-6.279$ & 0.033 & & 125 & 0.77 & $0.483-1.229$ & 0.274 & 0.69 & $0.428-1.112$ & 0.128 \\
\hline \multicolumn{17}{|l|}{ Gender } \\
\hline female & 1 & 77 & & & & & & & 1 & 77 & & & & & & \\
\hline male & & 93 & 1.737 & $0.963-3.131$ & 0.066 & 1.346 & $0.649-2.795$ & 0.425 & & 93 & 1.779 & $1.147-2.760$ & 0.010 & 1.245 & $0.704-2.200$ & 0.451 \\
\hline \multicolumn{17}{|l|}{ Smoking } \\
\hline no & 1 & 113 & & & & & & & 1 & 113 & & & & & & \\
\hline yes & & 57 & 1.718 & $0.921-3.207$ & 0.089 & 1.218 & $0.552-2.684$ & 0.626 & & 57 & 1.832 & $1.179-2.846$ & 0.007 & 1.364 & $0.761-2.445$ & 0.297 \\
\hline \multicolumn{17}{|l|}{ Pathology } \\
\hline ADC & 1 & 145 & & & & & & & 1 & 145 & & & & & & \\
\hline non-ADC & & 25 & 1.349 & $0.604-3.017$ & 0.465 & 1.548 & $0.680-3.520$ & 0.298 & & 25 & 1.59 & $0.894-2.826$ & 0.114 & 1.396 & $0.776-2.508$ & 0.265 \\
\hline \multicolumn{17}{|l|}{ Stage } \\
\hline IIIB & 1 & 9 & & & & & & & 1 & 9 & & & & & & \\
\hline IV & & 161 & 1.199 & $0.370-3.890$ & 0.762 & 1.030 & $0.291-3.650$ & 0.963 & & 161 & 1.627 & $0.594-4.455$ & 0.344 & 0.822 & $0.284-2.382$ & 0.719 \\
\hline \multicolumn{17}{|l|}{ EGFR } \\
\hline wild type & 1 & 71 & & & & & & & 1 & 71 & & & & & & \\
\hline mutation & & 99 & 0.485 & $0.271-0.868$ & 0.015 & 0.499 & $0.264-0.942$ & 0.032 & & 99 & 0.365 & $0.237-0.562$ & $<0.001$ & 0.419 & $0.262-0.672$ & $<0.001$ \\
\hline \multicolumn{17}{|l|}{ EGFR-TKI } \\
\hline 1st line & 1 & 83 & & & & & & & 1 & 83 & & & & & & \\
\hline$\geq 2$ nd line & & 87 & 1.338 & $0.751-2.384$ & 0.323 & 1.562 & $0.839-2.906$ & 0.159 & & 87 & 1.941 & $1.257-2.996$ & 0.003 & 1.756 & $1.099-2.806$ & 0.019 \\
\hline \multicolumn{17}{|l|}{ PD-L1 } \\
\hline negative & 1 & 58 & & & & & & & 1 & 58 & & & & & & \\
\hline positive & & 112 & 1.472 & $0.776-2.792$ & 0.236 & 1.901 & $0.953-3.790$ & 0.068 & & 112 & 1.003 & $0.641-1.569$ & 0.990 & 1.315 & $0.831-2.080$ & 0.242 \\
\hline
\end{tabular}

Abbreviations: OS, overall survival; PFS, progression-free survival; HR, hazard ratio; 95\%CI, 95\% confidence intervals; ADC, adenocarcinoma; non-ADC, non-adenocarcinoma; EGFR,

epidermal growth factor receptor; EGFR-TKI, EGFR-tyrosine kinase inhibitor; PD-L1, programmed cell death-ligand 1.

Non-small cell lung cancer patients using EGFR-TKIs at Sun Yat-sen University

Cancer Center (Guangzhou, China) during January 2008 to March 2014 (n=3543)

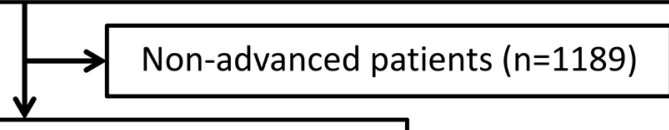

Advanced patients using EGFR-TKIs ( $\mathrm{n}=2354)$

Patients with unknown EGFR mutation status $(n=1586)$

Advanced patients with known EGFR mutation status using EGFR-TKIs ( $n=768)$

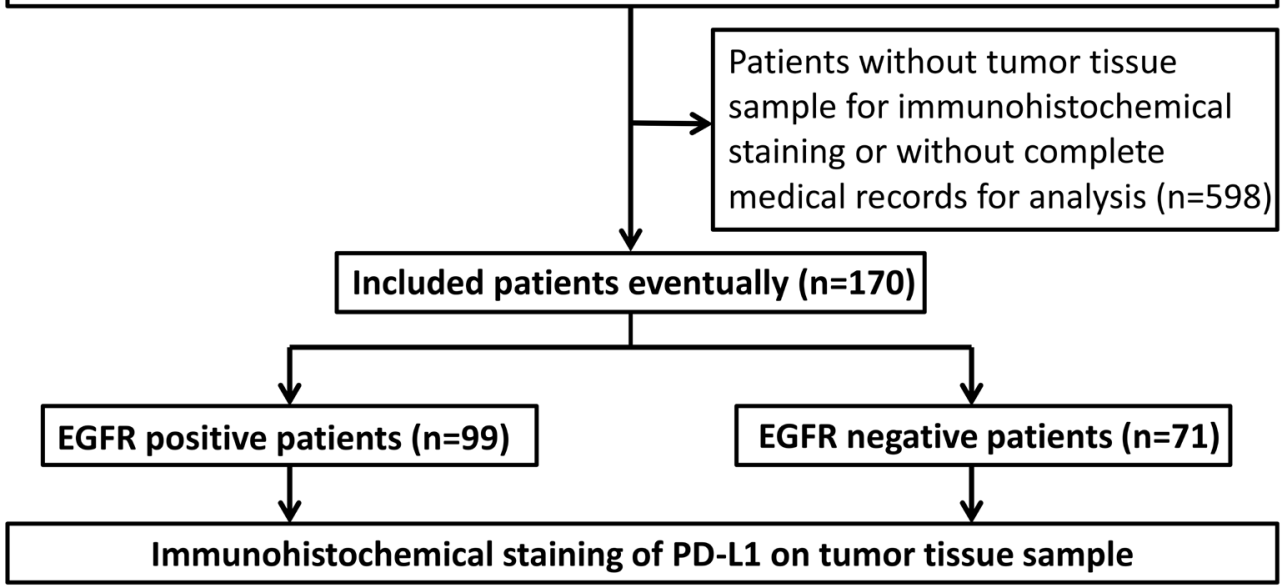

Figure 1: Flowing chart of the enrollment. Abbreviations: EGFR, epidermal growth factor receptor; EGFR-TKIs, EGFR-tyrosine kinase inhibitors; PD-L1, programmed cell death-ligand 1. 
A

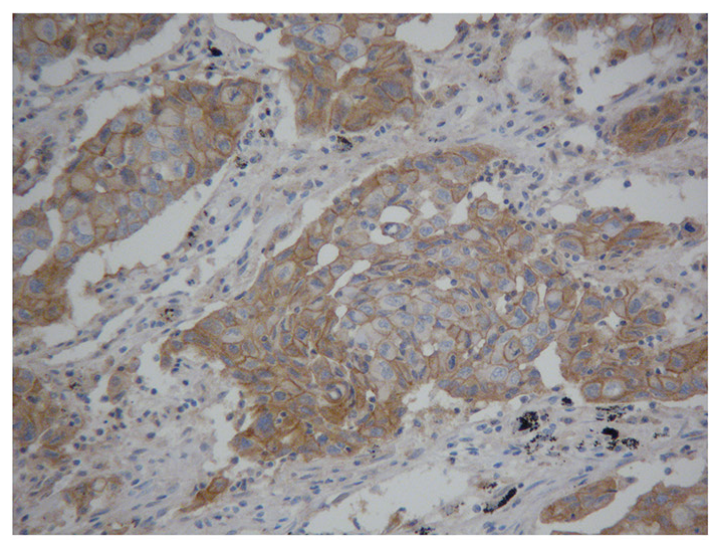

B

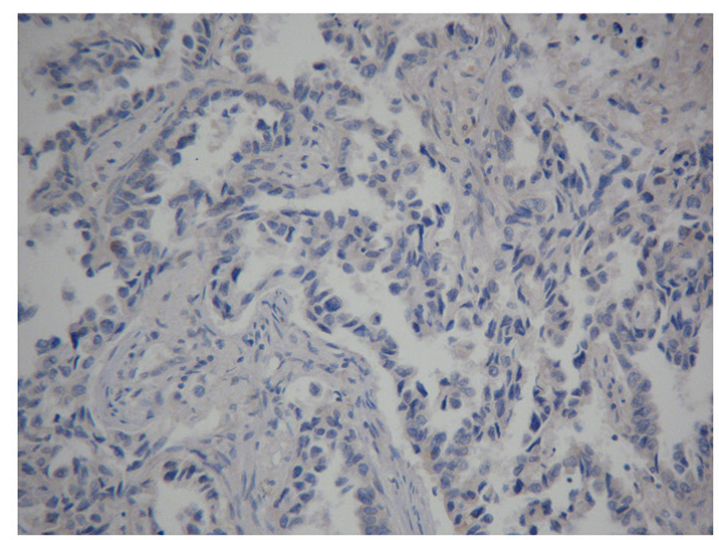

Figure 2: (A) Positive programmed cell death-ligand 1 (PD-L1) immunohistochemical staining with a membranous pattern. (B) Negative PD-L1 immunohistochemical staining. Original magnification, $20 \times$.
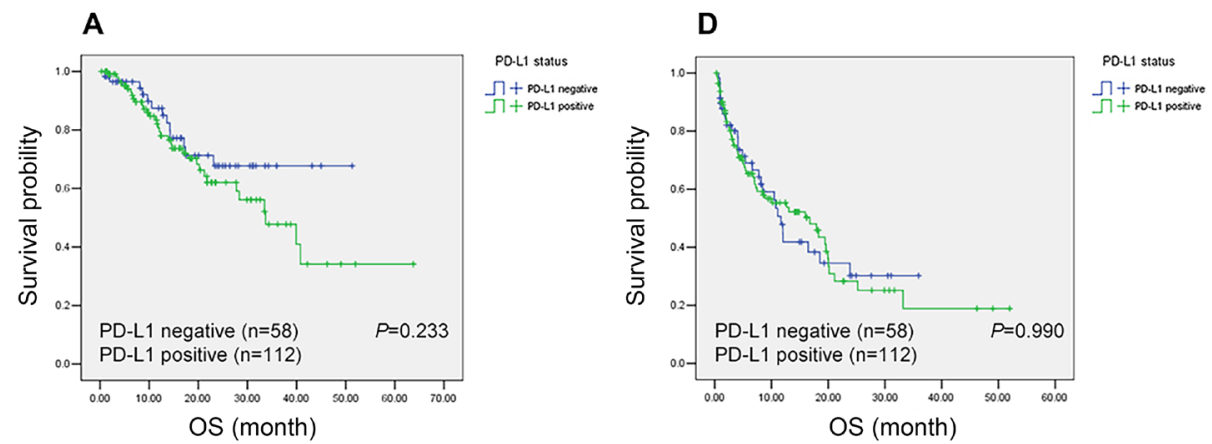

B
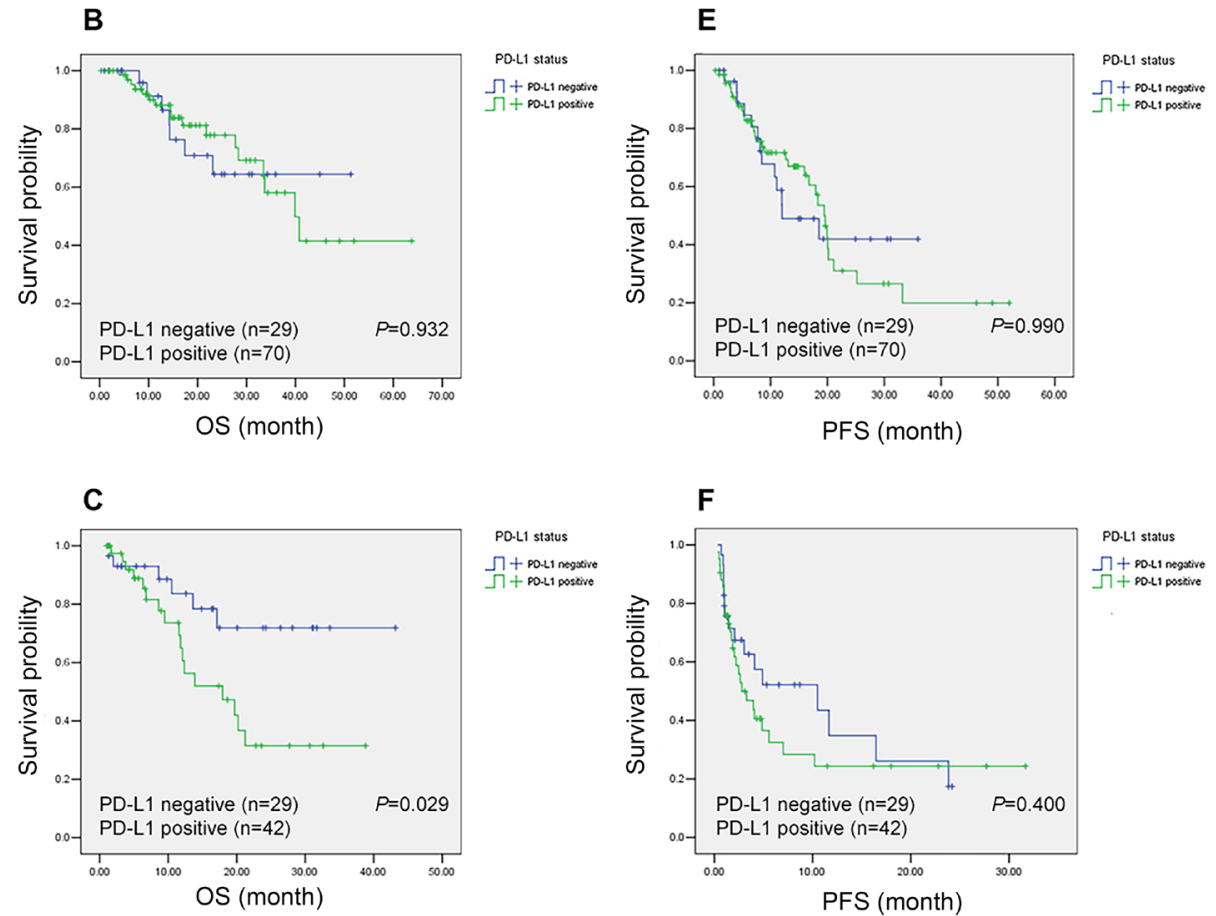

Figure 3: Kaplan-Meier curves of overall survival (OS) and progression-free survival (PFS) according to programmed cell death-ligand 1 (PD-L1) expression status in non-small cell lung cancer (NSCLC) patients. The P value for the difference between the two curves was determined by the log-rank test. Notes: (A) OS for overall population. (B) OS for patients with EGFR mutation. (C) OS for patients with EGFR wild type. (D) PFS for overall population. (E) PFS for patients with EGFR mutation. (F) PFS for patients with EGFR wild type. 
expression [2, 16, 18], and found PD-L1 was significantly higher in patients with the following characteristics: women, never smokers and with adenocarcinoma $[2,16]$. All these characteristics are hallmarks of EGFR mutations. In present study, we also found a borderline association between high PD-L1 expression and EGFR mutation in lung adenocarcinoma, while no significant relationship between ages, gender, smoking history and PD-L1 expression was found in NSCLC patients. The difference between the present and previous studies may be due to several reasons. First, the sample size varied among different studies and all data were retrospectively collected, resulting in potential bias. Second, the heterogeneity of baseline characteristics among these studies had also affected their outcomes, such as pathological stage and geographical distribution. Third, the threshold of positive PD-L1 expression was also different from each other in these studies. Fourth, the specificity and reproducibility of the commercially available antibodies and variations in Immunohistochemical technique weren't thoroughly assessed [15]. Thus, for future studies, more efforts to standardize a quantitative assay for PD-L1 expression are warranted.

In EGFR wild-type lung cancer, immune evasion induced by PD-L1 played an important role. PD-1/PDL1 pathway has been recognized as a key mechanism of immune evasion. Cancer cells can evade host immune systems by expressing certain ligands to down-regulate

A

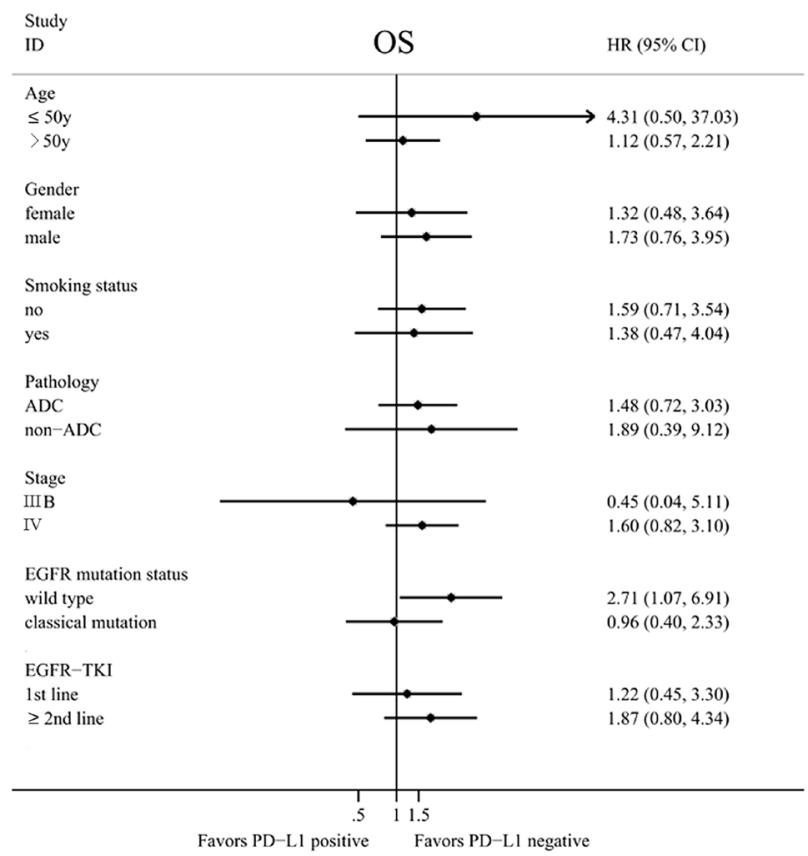

cytotoxic $\mathrm{T}$ lymphocytes through inhibitory pathways, which are usually initiated by ligand-receptor interactions [19] . PD-1 is one immune checkpoint expressed on the surface of T-cells upon activation [20]. PD-L1 is the major ligand for PD-1 and is expressed in various type of cancers [9] .PD-1/PD-L1 interaction is regarded as an inhibitory checkpoint for T-cell activation at its initial stage. In the present report, our data revealed that high PD-L1 expression was correlated with poor prognosis in EGFR wild-type patients but not in EGFR mutant population. This finding indicated that EGFR wild-type NSCLC failed to be controlled by the immune system which is inhibited by PD-L1 mediated antitumor activity. Then cancer cells can evade host immune responses by expressing PD-L1 to down-regulate T-cell activation in tumorous microenvironment [9], allowing cancer cells to survive and progress. Therefore, PD-L1 status was a significant prognostic factor for patients with EGFR wild type. This implicated that for this subset of population (EGFR wild-type and PD-L1 over-expression), PD-L1 blocker may be an alternative therapeutic strategy. For future clinical applications, more evidences to verify the feasibility are warranted.

According to previous studies, we could explain why patients with positive for PD-L1 expression tended to have better OS than PD-L1 negative ones in EGFR mutant NSCLC patients. The most possible reason was the cross interaction between EGFR pathway and PD-L1.

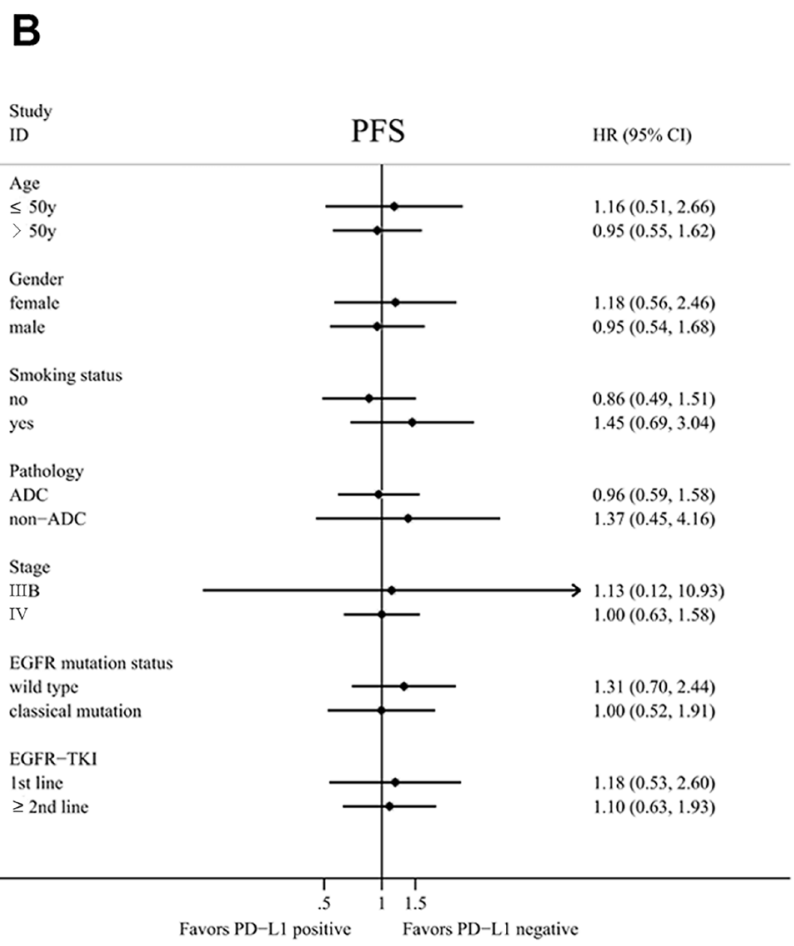

Figure 4: Forest plot of overall survival (OS) and progression-free survival (PFS) according to programmed cell death-ligand 1 (PD-L1) expression status in subgroup analysis. Abbreviations: HR, hazard ratio, HR $<1$ implies a lower risk of progression or death for patients; $95 \%$ CI, $95 \%$ confidence intervals. 
A study by Azuma K et al. [2] found that inhibition of EGFR signaling with erlotinib led to down-regulation of the expression of PD-L1 in EGFR mutant NSCLC cells but not in those with wild-type EGFR, indicating that the expression of PD-L1 might be dependent on EGFR signaling conferred by activating EGFR mutations. In another research, Akbay EA et al. [16] also found that PDL1 expression was reduced by EGFR inhibitors in NSCLC cell lines with activated EGFR. Therefore, for EGFR mutant NSCLC patients, EGFR-TKIs could perform a dual therapeutic response. The down-regulation of PD-L1 expression and the consequent restoration of an antitumor immune response might contribute to the durable therapeutic response. EGFR activation up regulated PDL1 through MAPK signaling pathway. As previously reported, EGFR remarkably increased the activity of ERK1/2 and AKT which are involved in the proliferation, anti-apoptosis, and invasion of tumor cells [21, 22]. Chen $\mathrm{N}$ et al. [23] further demonstrated that the up-regulation of PD-L1 mediated by EGFR activation was associated with the activation of ERK1/2/c-Jun. By inhibiting p-ERK1/2, PD-L1 decreased following p-ERK1/2/p-Jun downregulation in a clear dose-dependent manner [23]. This detailed mechanism confirmed that EGFR mutant NSCLC patients may benefit not only from direct tumor killing effect of EGFR-TKIs but also indirectly from immune enhancement after EGFR-TKIs treatment.

As we known, targeted therapy usually have rapid and impressive response rates but modest progression-free survival while immunotherapy can achieve durable tumor control but associated with lower response rates [24]. To address this, investigators have proposed combining these strategies. There is a scientific rationale supporting the combination of targeted therapy and immunotherapy [25]. For patients with melanoma, the concept of potential synergy with BRAF-targeted therapy and immunotherapy is being empirically investigated in clinical trials [26-28]; however, much remains to be learned. Response data from these initial trials are not mature, and additional trials will be needed to determine the appropriate sequence, schedule, and duration of therapy if there is evidence of synergy. Yet, there were no data about the combinatorial strategies of EGFR-TKIs with anti-PD-1/PD-L1. While there were no direct verified evidences, this combining therapy may be an alternative strategy in EGFR mutant NSCLC. In the future, more well-designed in vitro and vivo studies to explore molecular mechanisms of combining EGFR-TKIs and anti-PD-1/PD-L1 antibodies are urgently required. Randomized clinical trials to instruct how best to combine therapeutic agents are also needed.

Currently, though gefitinib and erlotinib are regarded as the first line treatment of classical EGFR mutant NSCLC patients, a majority of them eventually develop secondary resistance to gefitinib and erlotinib. Previous treatment options for EGFR-TKIs resistance include CO1686 [29], AZD9291 [30] and HM61713 [31] for EGFR
T790M and EGFR-TKIs plus c-met inhibitors for c-met amplification [32]. However, the role of immunotherapy in EGFR-TKIs-resistant patients has not been revealed. Chen $\mathrm{N}$ et al. [23] demonstrated that the protein level of PD-L1 in EGFR-mutant NSCLC cell lines (PC-9, HCC827 and H1975) was significantly higher than that in EGFR-wild type cell lines (A539, H1993). Moreover, the expression of PD-L1 was the highest in resistant cells (H1975 cells, with EGFR-T790M mutation). Anti- PD-1/ PD-L1 axis could significantly decrease the viability of gefitinib resistant $\mathrm{H} 1975$ cells. This implied that blockade of PD-1/PD-L1 might be a promising optional treatment for NSCLC patients with EGFR mutation, especially for EGFR-TKIs resistant NSCLC patients. Future clinical studies are needed to test the feasibility.

Notably, this is the first study to assess the relationship between PD-L1 expression and prognosis as well as efficacy in Chinese advanced NSCLC patients with treatment of EGFR-TKIs. We prove that high PDL1 expression is likely to be associated with the presence of EGFR mutation in advanced lung adenocarcinoma. Moreover, PD-L1 over-expression can be considered as a poor prognostic indicator of OS in EGFR wild-type patients treated with EGFR-TKIs.

\section{PATIENTS AND METHODS}

\section{Patients}

A total of 3543 consecutive NSCLC patients who have taken oral EGFR-TKIs at Sun Yat-sen University Cancer Center (Guangzhou, China) from January 2008 to March 2014 were screened in the study. Patients were recruited if they met the following conditions: 1, treated with EGFR-TKIs; 2, stage 3b/4 NSCLC or recurrent disease after surgery or chemotherapy; 3, with known EGFR mutational status; 4, having detailed medical records and had enough tumor tissue samples for immunohistochemical staining of PD-L1. Finally, a total of 170 patients were eligible. Figure 1 summarized the process of patients' selection. Baseline clinical and pathological features were collected, as well as tissue specimens from surgery or biopsy. The clinicopathological features of the patients included age, gender, smoking status, pathological type, Union for International Cancer Control (UICC) stage (the seventh edition), EGFR mutation status and EGFR-TKI treatment history. Fifty years old was chosen as the cutoff for dividing the high or low age group (50y was low and 50y was high, respectively). Smoking history was noted as yes or no (no-smoking refers to patients who had never smoked in their lifetime). Pathological subtype was divided into adenocarcinoma or non- adenocarcinoma. All patients were restaged according to the seventh edition of UICC 
Staging System for NSCLC. EGFR exon 19 deletions or exon 21 base substitutions were considered as classical EGFR mutations by fluorescent quantitative polymerase chain reaction (PCR). The study was approved by the Institutional Review Board of Sun Yat-Sen University Cancer Center (Guangzhou, China). All the patients had provided written informed consent before samples were collected.

\section{Immunohistochemistry analyses}

Immunohistochemical staining was performed using rabbit monoclonal anti-human antibody (E1L3NTM, Cell Signaling Technology, Danvers, MA, 1:200) for testing the expression of PD-L1 in human NSCLC specimens. Five- $\mu \mathrm{m}$-thick Sections were cut from the formalin-fixed, paraffin-embedded (FFPE) tumor block and then routinely deparaffined and rehydrated. For antigen retrieval, slides were heated in a microwave oven for 30 minutes in citrate buffer solution $(\mathrm{pH}=7.4)$ and cooled slowly at room temperature for 20 minutes. After blocking the activity of endogenous peroxidase with $3 \%$ hydrogen peroxide for 8 minutes, the sections were treated with primary antibodies and incubated for overnight (more than 12 hours). Subsequently, the slides were rinsed in PBS three times and incubated in HRR-linked secondary antibodies. After incubation, slides were washed again with PBS and then visualized using diaminobenzidine. Finally, Mayer's hematoxylin was used to counterstain the sections, which were then dehydrated and mounted.

Two pathologists who were blinded to the clinical or pathological information of these patients independently assess the expression of PD-L1. Semiquantitative H score (maximum value of 300 corresponding to $100 \%$ of tumor cells positive for PD-L1 with an overall staining intensity score of 3) was defined as multiplying the percentage of stained cells by an intensity score ( 0 , absent; 1 ,weak; 2 , moderate; and 3, strong). A 5\% proportion of membranepositive tumor cells which were defined as H-score $\geq 5$ have been used as cutoff for PD-L1 positivity [33, 34], as this cut-point is reported to be associated with clinical response to anti-PD-1 therapy [34].

\section{Statistical analysis}

All the statistical analysis was performed using SPSS 20.0 for Windows (IBM, Armonk, NY). The cut-off value of age were obtained by X-tile software (Version 3.6.1, Yale University, New Haven, CT), taking clinical expertise into consideration. Pearson's chi-squared test or continuity correction test was used to assess correlations between PD-L1 expression and clinicopathologic variables. Univariate and multivariate logistic regression analysis were used to test the association between PD-L1 expression and EGFR-TKIs' efficacy (ORR and DCR).
OS was defined as the time from diagnosis to the end of the follow-up (August 2014). And PFS was the time from beginning to taking TKI to recurrence or last follow-up. OS and PFS analyses were estimated with Kaplan-Meier method and multivariable analyses were performed to assess survival difference. The results of ORR and DCR were reported with OR and its $95 \%$ CI, while prognostic results were reported with $\mathrm{HR}$ and its $95 \% \mathrm{CI}$. OR $>1$ indicated that EGFR-TKI was more effective in PDL1 positive patients. HR $<1$ implied a lower risk of progression or death for patients with positive expression of PD-L1. A two sided p-value of $<0.05$ was considered statistically significant.

\section{ACKNOWLEDGEMENTS}

This study was supported by the following funds:

1. National High Technology Research and Development Program of China (Grant No. 2012AA02A502)

2. Innovative drug $R \& D$ center based on real-time high-throughput cell-based screening platform and large capacity compound library (Grant No. 2013ZX09401003002)

3. National Natural Science Funds of China (Grant No. 81372502 and 81201917 )

4. Natural Science Foundation of Guangdong (Grant No. S2013010016564),

5. Specialized Research Fund for the Doctoral Program of Higher Education (20120171120116),

6. Young Teacher Training Program of Sun Yat-Sen University (14ykpy38),

7. Outstanding Young Talent Cultivation Project of Sun Yat-Sen University Cancer Center (04140701)

8. Wu Jieping Medical Foundation Project (Grant No. 320.6750.131)

All the funders had no role in study design, data collection and analysis, decision to publish, or preparation of the manuscript.

\section{DISCLOSURE}

All authors have no conflicts of interest.

\section{REFERENCES}

1. Siegel R, Ma J, Zou Z and Jemal A. Cancer statistics, 2014. CA: a cancer journal for clinicians. 2014; 64:9-29.

2. Azuma K, Ota K, Kawahara A, Hattori S, Iwama E, Harada T, Matsumoto K, Takayama K, Takamori S, Kage M, Hoshino T, Nakanishi Y and Okamoto I. Association of PD-L1 overexpression with activating EGFR mutations in surgically resected nonsmall-cell lung cancer. Annals of oncology : official journal of the European Society for Medical Oncology / ESMO. 2014; 25:1935-1940. 
3. Oxnard GR, Binder A and Janne PA. New targetable oncogenes in non-small-cell lung cancer. Journal of clinical oncology : official journal of the American Society of Clinical Oncology. 2013; 31:1097-1104.

4. Shigematsu H, Lin L, Takahashi T, Nomura M, Suzuki M, Wistuba, II, Fong KM, Lee H, Toyooka S, Shimizu N, Fujisawa T, Feng Z, Roth JA, Herz J, Minna JD and Gazdar AF. Clinical and biological features associated with epidermal growth factor receptor gene mutations in lung cancers. Journal of the National Cancer Institute. 2005; 97:339-346.

5. Jorge SE, Kobayashi SS and Costa DB. Epidermal growth factor receptor (EGFR) mutations in lung cancer: preclinical and clinical data. Brazilian journal of medical and biological research $=$ Revista brasileira de pesquisas medicas e biologicas / Sociedade Brasileira de Biofisica [et al]. 2014; 47:929-939.

6. Agata Y, Kawasaki A, Nishimura H, Ishida Y, Tsubata T, Yagita $\mathrm{H}$ and Honjo T. Expression of the PD-1 antigen on the surface of stimulated mouse $\mathrm{T}$ and $\mathrm{B}$ lymphocytes. International immunology. 1996; 8:765-772.

7. Nishimura $\mathrm{H}$ and Honjo T. PD-1: an inhibitory immunoreceptor involved in peripheral tolerance. Trends in immunology. 2001; 22:265-268.

8. Watanabe N, Gavrieli M, Sedy JR, Yang J, Fallarino F, Loftin SK, Hurchla MA, Zimmerman N, Sim J, Zang X, Murphy TL, Russell JH, Allison JP and Murphy KM. BTLA is a lymphocyte inhibitory receptor with similarities to CTLA-4 and PD-1. Nature immunology. 2003; 4:670679.

9. Sznol M and Chen L. Antagonist antibodies to PD-1 and B7-H1 (PD-L1) in the treatment of advanced human cancer. Clinical cancer research : an official journal of the American Association for Cancer Research. 2013; 19:1021-1034.

10. Blank C, Brown I, Peterson AC, Spiotto M, Iwai Y, Honjo T and Gajewski TF. PD-L1/B7H-1 inhibits the effector phase of tumor rejection by $\mathrm{T}$ cell receptor (TCR) transgenic CD8+ T cells. Cancer research. 2004; 64:1140-1145.

11. Keir ME, Liang SC, Guleria I, Latchman YE, Qipo A, Albacker LA, Koulmanda M, Freeman GJ, Sayegh MH and Sharpe AH. Tissue expression of PD-L1 mediates peripheral $\mathrm{T}$ cell tolerance. The Journal of experimental medicine. 2006; 203:883-895.

12. Haspot F, Fehr T, Gibbons C, Zhao G, Hogan T, Honjo T, Freeman GJ and Sykes M. Peripheral deletional tolerance of alloreactive $\mathrm{CD} 8$ but not $\mathrm{CD} 4 \mathrm{~T}$ cells is dependent on the PD-1/PD-L1 pathway. Blood. 2008; 112:2149-2155.

13. Boland JM, Kwon ED, Harrington SM, Wampfler JA, Tang H, Yang P and Aubry MC. Tumor B7-H1 and B7-H3 expression in squamous cell carcinoma of the lung. Clinical lung cancer. 2013; 14:157-163.

14. Chen YY, Wang LB, Zhu HL, Li XY, Zhu YP, Yin YL, Lu FZ, Wang ZL and Qu JM. Relationship between programmed death-ligand 1 and clinicopathological characteristics in non-small cell lung cancer patients. Chinese medical sciences journal = Chung-kuo i hsueh k'o hsueh tsa chih / Chinese Academy of Medical Sciences. 2013; 28:147-151.

15. Velcheti V, Schalper KA, Carvajal DE, Anagnostou VK, Syrigos KN, Sznol M, Herbst RS, Gettinger SN, Chen L and Rimm DL. Programmed death ligand-1 expression in non-small cell lung cancer. Laboratory investigation; a journal of technical methods and pathology. 2014; 94:107116.

16. Akbay EA, Koyama S, Carretero J, Altabef A, Tchaicha JH, Christensen CL, Mikse OR, Cherniack AD, Beauchamp EM, Pugh TJ, Wilkerson MD, Fecci PE, Butaney M, Reibel JB, Soucheray M, Cohoon TJ, et al. Activation of the PD-1 pathway contributes to immune escape in EGFR-driven lung tumors. Cancer discovery. 2013; 3:1355-1363.

17. Zhang Y, Kang S, Shen J, He J, Jiang L, Wang W, Guo Z, Peng G, Chen G, He J and Liang W. Prognostic Significance of Programmed Cell Death 1 (PD-1) or PD-1 Ligand 1 (PDL1) Expression in Epithelial-Originated Cancer: A MetaAnalysis. Medicine. 2015; 94:e515.

18. D’Incecco A, Andreozzi M, Ludovini V, Rossi E, Capodanno A, Landi L, Tibaldi C, Minuti G, Salvini J, Coppi E, Chella A, Fontanini G, Filice ME, Tornillo L, Incensati RM, Sani S, et al. PD-1 and PD-L1 expression in molecularly selected non-small-cell lung cancer patients. British journal of cancer. 2015; 112:95-102.

19. Yang CY, Lin MW, Chang YL, Wu CT and Yang PC. Programmed cell death-ligand 1 expression in surgically resected stage I pulmonary adenocarcinoma and its correlation with driver mutations and clinical outcomes. European journal of cancer. 2014; 50:1361-1369.

20. Keir ME, Butte MJ, Freeman GJ and Sharpe AH. PD-1 and its ligands in tolerance and immunity. Annual review of immunology. 2008; 26:677-704.

21. Gadgeel SM and Wozniak A. Preclinical rationale for PI3K/ Akt/mTOR pathway inhibitors as therapy for epidermal growth factor receptor inhibitor-resistant non-small-cell lung cancer. Clinical lung cancer. 2013; 14:322-332.

22. McCubrey JA, Steelman LS, Chappell WH, Abrams SL, Wong EW, Chang F, Lehmann B, Terrian DM, Milella M, Tafuri A, Stivala F, Libra M, Basecke J, Evangelisti C, Martelli AM and Franklin RA. Roles of the Raf/MEK/ERK pathway in cell growth, malignant transformation and drug resistance. Biochimica et biophysica acta. 2007; 1773:12631284.

23. Chen N, Fang W, Zhan J, Hong S, Tang Y, Kang S, Zhang Y, He X, Zhou T, Qin T, Huang Y, Yi X and Zhang L. Up-regulation of PD-L1 by EGFR Activation Mediates the Immune Escape in EGFR-driven NSCLC: Implication for Optional Immune Targeted Therapy for NSCLC Patients with EGFR Mutation. Journal of thoracic oncology : official publication of the International Association for the Study of Lung Cancer. 2015.

24. Kim T, Amaria RN, Spencer C, Reuben A, Cooper ZA 
and Wargo JA. Combining targeted therapy and immune checkpoint inhibitors in the treatment of metastatic melanoma. Cancer biology \& medicine. 2014; 11:237-246.

25. Wargo JA, Cooper ZA and Flaherty KT. Universes collide: combining immunotherapy with targeted therapy for cancer. Cancer discovery. 2014; 4:1377-1386.

26. Wilmott JS, Long GV, Howle JR, Haydu LE, Sharma RN, Thompson JF, Kefford RF, Hersey P and Scolyer RA. Selective BRAF inhibitors induce marked T-cell infiltration into human metastatic melanoma. Clinical cancer research : an official journal of the American Association for Cancer Research. 2012; 18:1386-1394.

27. Cooper ZA, Juneja VR, Sage PT, Frederick DT, Piris A, Mitra D, Lo JA, Hodi FS, Freeman GJ, Bosenberg MW, McMahon M, Flaherty KT, Fisher DE, Sharpe AH and Wargo JA. Response to BRAF inhibition in melanoma is enhanced when combined with immune checkpoint blockade. Cancer immunology research. 2014; 2:643-654.

28. Ribas A, Hodi FS, Callahan M, Konto C and Wolchok J. Hepatotoxicity with combination of vemurafenib and ipilimumab. The New England journal of medicine. 2013; 368:1365-1366

29. Liao BC, Lin CC and Yang JC. Second and third-generation epidermal growth factor receptor tyrosine kinase inhibitors in advanced nonsmall cell lung cancer. Current opinion in oncology. 2015; 27:94-101.

30. Meador CB, Jin H, de Stanchina E, Nebhan CA, Pirazzoli V, Wang L, Lu P, Vuong H, Hutchinson KE, Jia P, Chen X, Eisenberg R, Ladanyi M, Politi K, Zhao Z, Lovly CM, et al. Optimizing the Sequence of Anti-EGFR-Targeted Therapy in EGFR-Mutant Lung Cancer. Molecular cancer therapeutics. 2015; 14:542-552.

31. Peters S, Zimmermann S and Adjei AA. Oral epidermal growth factor receptor tyrosine kinase inhibitors for the treatment of non-small cell lung cancer: comparative pharmacokinetics and drug-drug interactions. Cancer treatment reviews. 2014; 40:917-926.

32. Nakagawa $T$, Takeuchi S, Yamada T, Nanjo S, Ishikawa D, Sano T, Kita K, Nakamura T, Matsumoto K, Suda K, Mitsudomi T, Sekido Y, Uenaka T and Yano S. Combined therapy with mutant-selective EGFR inhibitor and Met kinase inhibitor for overcoming erlotinib resistance in EGFR-mutant lung cancer. Molecular cancer therapeutics. 2012; 11:2149-2157.

33. Petrelli NJ, Winer EP, Brahmer J, Dubey S, Smith S, Thomas C, Vahdat LT, Obel J, Vogelzang N, Markman M, Sweetenham JW, Pfister D, Kris MG, Schuchter LM, Sawaya R, Raghavan D, et al. Clinical Cancer Advances 2009: major research advances in cancer treatment, prevention, and screening--a report from the American Society of Clinical Oncology. Journal of clinical oncology : official journal of the American Society of Clinical Oncology. 2009; 27:6052-6069.

34. Topalian SL, Hodi FS, Brahmer JR, Gettinger SN, Smith DC, McDermott DF, Powderly JD, Carvajal RD, Sosman
JA, Atkins MB, Leming PD, Spigel DR, Antonia SJ, Horn L, Drake CG, Pardoll DM, et al. Safety, activity, and immune correlates of anti-PD-1 antibody in cancer. The New England journal of medicine. 2012; 366:2443-2454. 